\title{
JUSTIFICACIÓN DEL ATLETISMO COMO CONTENIDO CURRICULAR DE PRIMARIA BASÁNDOSE EN UNA PROPUESTA PRÁCTICA
}

\section{RESUMEN}

El atletismo como contenido del área de Educación Física en Educación Primaria puede ser cuestionado al no estar incluido como bloque de contenido y por tener una orientación que no responda a los objetivos y criterios metodológicos que implican dicha etapa educativa. Haciendo uso de los documentos oficiales emitidos por el Ministerio de Educación y Ciencia y de las aportaciones de autores especialistas en el campo de la iniciación deportiva, se justifica su inclusión desde los diferentes elementos curriculares, siempre y cuando contemple una serie de orientaciones metodológicas tales como darle un enfoque globalizador y emplear la actividad lúdica como principal recurso, finalizando este artículo, a modo de ejemplo esclarecedor, con una unidad didáctica.

\section{Introducción}

Reconocido en todo el mundo como deporte rey, el atletismo es el más universal y ancestral de los deportes conocidos hasta nuestros días (Zurita, 1998). No necesita de complejas instalaciones ni de enrevesados instrumentos tecnológicos para su sencilla puesta en práctica: basta con un espacio abierto donde se puedan realizar diferentes tipos de lanzamientos, saltos y carreras. Por ello, no es de extrañar que se trate de una práctica deportiva con gran arraigo en la educación y en la cultura tanto de países occidentales como de orientales.

Desde principios del siglo $X X$, el deporte $y$, por consiguiente, el atletismo como actividad deportiva individual, se ha constituido como uno de los contenidos más extendidos del currículum de la Educación Física Escolar, circunstancia que se da en la práctica totalidad de los países. Sin embargo, el tratamiento que recibe no es homogéneo, existiendo ciertas contradicciones entre la tradición y la nueva concepción que, desde los planteamientos de la reforma educativa, pugna por hacerse un lugar en las escuelas y entre sus profesores (De la Torre y Hernández, 1996).
ALFONSO VALERO VALENZUELA. EE.PP. SAGRADA FAMILIA DE ÚBEDA (JAEN).

JOSÉLUIS CONDE CAVEDA UNIVERSIDAD DE GRANADA. 
Junto a este hecho, numerosos autores, entre otros Rodríguez (1993), y Castejón y López (1997), han analizado los Reales Decretos, llegando todos a la conclusión de que el deporte como contenido educativo tiene cabida dentro de la Educación Primaria, concretamente dentro del bloque de contenidos de Juegos en Educación Física. Por tanto, el atletismo como deporte que es, también puede ser seleccionado como contenido a tratar dentro del bloque de Juegos con el que lograr alcanzar los objetivos generales del área, haciendo que el alumno conozca la verdadera raíz social que encierra el atletismo y las conductas humanas que a través del mismo se pueden adquirir.

Pasando a la segunda duda planteada acerca de cuál es el enfoque que se le ha de dar a la iniciación al atletismo, básicamente ésta se puede plantear desde dos perspectivas totalmente diferentes; desde un punto de vista de rendimiento, donde los aprendizajes están enfocados hacia la consecución de unos logros. Y un segundo punto de vista, donde la iniciación al atletismo es medio para la consecución del objetivo de la formación integral del alumno, fomentando valores de cooperación, solidaridad y respeto a los demás, y siendo los juegos y la técnica deportiva elementos empleados para su alcance.

Es este segundo punto de vista desde el que hay que enfocar la iniciación atlética, es decir, desde una concepción educativa, donde el eje del proceso de enseñanza - aprendizaje sea el alumno, siendo el atletismo el medio para la consecución de los objetivos generales de etapa. Continuando con el análisis del Diseño Curricular de Educación Primaria elaborado por el M.E.C. (1991b; pg. 4115), resalta una declaración de intenciones muy en esta línea y que indica que "en Educación Física se ha de llevar una educación a través del cuerpo y el movimiento y ésta no se ha de reducir exclusivamente a aspectos perceptivos y motrices, sino que ha de implicar otros de carácter expresivo, comunicativo, afectivo y cognitivo».

Una referencia más al M.E.C. (1991b, pgs. 4115-4116), nos viene a decir que la "práctica deportiva tal y como es apreciada en la sociedad se corresponde con planteamientos competitivos selectivos que no siempre son compatibles con las intenciones educativas del currículo", confirmando que el enfoque que los profesionales de la educación han de darle a esta iniciación atlética ha de ser el de una formación integral del niño, donde se fomente el desarrollo de capacidades cognitivas, relacionales, emocionales y expresivas, dejando de lado la finalidad última de obtener un resultado en la actividad competitiva.

Con esto, no se debe buscar la exclusión de la competición en la iniciación deportiva, pues como bien apunta Arnold (1991), la competición es inherente al deporte y puede utilizarse con un fin educativo siempre que se cumpla con algunas premisas, siendo las más importantes, huir de la eliminación (Castejón, 1997), ser un medio socializador importante y estar al servicio del alumnado para darle a conocer reglas y normas (Giménez, 1997).

Por tanto, los argumentos anteriores junto con el análisis del Decreto de Enseñanzas Mínimas del Ministerio de Educación y Ciencia (M.E.C., 1991b), referidos a los elementos curriculares de la Educación Física en Primaria, van a ser los elementos claves que nos ayuden a esclarecer la idoneidad o no de la iniciación al atletismo en la etapa de la Educación Primaria

\section{Justificación del atletismo teniendo en cuenta los elemen- tos curriculares de la educación física en primaria}

Los elementos integrantes del currículum; objetivos, contenidos, metodología y criterios de evaluación (M.E.C., 1991b), son los que se van a tener en cuenta para realizar la justificación del atletismo en la etapa de la Educación Primaria, relacionando en este artículo cada uno de los elementos con la práctica atlética, estableciendo vínculos directos y aportando ejemplos clarificadores.

\section{Los objetivos}

Concebidos como la meta a alcanzar, gracias a la cuál se contribuye fundamentalmente al desarrollo de las capacidades de comunicación, pensamiento lógico y conocimiento del entorno social y natural de los alumnos, debemos distinguir entre generales de etapa y de área, entendiendo Díaz Lucea (1995), por objetivos de etapa; enunciados o intenciones educativas que fijan las capacidades que los alumnos y alumnas habrán de adquirir, basándose en las diferentes áreas al final de la etapa correspondiente. Mientras que objetivos de área son enunciados que fijan las capacidades que los alumnos habrán de adquirir a través de esa área concreta y al finalizar la etapa educativa correspondiente.

Con la intención de ejemplificar mediante planteamientos lúdicos de iniciación al atletismo cómo pueden alcanzarse los objetivos de área, y por ende, probar que el atletismo tiene cabida dentro del área de Educación Física, y contribuye a la consecución de los objetivos, se ha elaborado un cuadro de triple entrada (cuadro 1), donde en una columna se enuncian los objetivos del área, en la siguiente columna, el nivel de significatividad que posee la iniciación al atletismo con dicho objetivo, y en la tercera columna, un ejemplo de propuesta educativa con el que se logra dicho objetivo.

Estos objetivos de área contemplados en el cuadro 1 , a su vez se concretan en objetivos de ciclo y dentro de éstos para cada bloque de contenidos, de tal forma que se detallan con un grado de especificidad y concreción cada vez mayor, llegando al máximo nivel de concreción por medio de los objetivos didácticos, que serían los más precisos, viniendo a referirse a cada unidad didáctica.

Con objeto de aclarar estas ideas y esquematizar paso a paso como los objetivos de los diferentes niveles de concreción pueden dar paso a los de una unidad didáctica de iniciación al atletismo se ha elaborado el cuadro 2 , donde únicamente se han dejado reflejados aquellos que se consideran más relevantes o definitorios, no olvidando su carácter orientativo y evitando entrar en polémicas de si los objetivos señalados son los más indicados para tal caso. 


\section{Los contenidos}

Tradicionalmente los contenidos se han presentado cargados de significación intelectualista, mientras que en la actualidad se tiende a una acepción mucho más amplia que abarca las finalidades de la escolaridad (Contreras, 1998).

\begin{tabular}{|c|c|c|}
\hline $\begin{array}{l}\text { OBJETIVOS DEL ÁREA DE } \\
\text { EDUCACIÓN FÍSICA }\end{array}$ & $\begin{array}{l}\text { SIG } \\
(*)\end{array}$ & $\begin{array}{l}\text { EJEMPLO DE PROPUESTA EDUCATIVA } \\
\text { PARA LA INICIACIÓN AL ATLETISMO }\end{array}$ \\
\hline $\begin{array}{l}\text { 1. Conocer y valorar su cuerpo y la actividad } \\
\text { física como medio de exploración y disfrute } \\
\text { de sus posibilidades motrices, de relación } \\
\text { con los demás y como recurso para organizar } \\
\text { el tiempo libre. }\end{array}$ & & $\begin{array}{l}\text { Las } 4 \text { esquinas. Grupos de } 5 \text {, formando un cuadrado con } \\
\text { un compañero en el centro. El juego consistirá en ir } \\
\text { cambiando de esquinas los compañeros, haciendo } \\
\text { skipping por delante sin que el jugador del centro le dé } \\
\text { tiempo a ocupar una libre (haciendo skipping por } \\
\text { detrás). }\end{array}$ \\
\hline $\begin{array}{l}\text { 2. Adoptar hábitos de higiene, de } \\
\text { alimentación, de posturas y de ejercicio } \\
\text { físico, manifestando una actitud responsable } \\
\text { hacia su propio cuerpo y de respeto a los } \\
\text { demás, relacionando estos hábitos con los } \\
\text { efectos sobre la salud. }\end{array}$ & & $\begin{array}{l}\text { Realizar una carrera de aproximación para saltar con } \\
\text { un pie sobre un trampolín y caer con los dos pies al } \\
\text { foso de longitud, evitando caer con los glúteos o con un } \\
\text { solo pie. Una vez realizado el salto se pasará a nivelar } \\
\text { la arena del foso con un rastrillo. }\end{array}$ \\
\hline $\begin{array}{l}\text { 3. Regular y dosificar su esfuerzo llegando a } \\
\text { un nivel de autoexigencia acorde con sus } \\
\text { posibilidades y la naturaleza de la tarea que } \\
\text { se realiza, utilizando como criterio } \\
\text { fundamental de valoración dicho esfuerzo y } \\
\text { no el resultado obtenido. }\end{array}$ & & $\begin{array}{l}\text { Competición de relevos por equipos, donde se habrá de } \\
\text { ir pasando un testigo con la técnica de entrega "de } \\
\text { abajo - arriba". Ganará el juego quien mejor ejecute la } \\
\text { entrega del testigo, siendo secundario quién lo realice } \\
\text { antes. }\end{array}$ \\
\hline $\begin{array}{l}\text { 4. Resolver problemas que exijan el dominio } \\
\text { de patrones motrices básicos adecuándose a } \\
\text { los estímulos perceptivos y seleccionando los } \\
\text { movimientos, previa valoración de sus } \\
\text { posibilidades. }\end{array}$ & ++ & $\begin{array}{l}\text { Competición de salto de altura, donde habrán de } \\
\text { cruzar entre dos gomas elásticas, dejando una por } \\
\text { debajo y la otra por encima del cuerpo. Ir aumentando } \\
\text { la altura. }\end{array}$ \\
\hline $\begin{array}{l}\text { 5. Utilizar sus capacidades físicas básicas y } \\
\text { destrezas motrices y su conocimiento de la } \\
\text { estructura y funcionamiento del cuerpo para } \\
\text { la actividad física y para adaptar el } \\
\text { movimiento a las circunstancias y } \\
\text { condiciones de cada situación. }\end{array}$ & & $\begin{array}{l}\text { Marchando por el espacio, sólo cuando el profesor diga } \\
\text { un componente del aparato locomotor, los niños } \\
\text { acelerarán su marcha para buscar al resto de los } \\
\text { componentes de su grupo y formar la inicial de la } \\
\text { palabra que haya dicho el profesor. Por ejemplo, } \\
\text { tendrán que formar la "H", cuando mencione la palabra } \\
\text { ihuesos!. }\end{array}$ \\
\hline $\begin{array}{l}\text { 6. Participar en juegos y actividades } \\
\text { estableciendo relaciones equilibradas y } \\
\text { constructivas con los demás, evitando la } \\
\text { discriminación por características } \\
\text { personales, sexuales y sociales, así como los } \\
\text { comportamientos agresivos y las actitudes } \\
\text { de rivalidad en las actividades competitivas. }\end{array}$ & +++ & $\begin{array}{l}\text { Competición de martillos. Grupos de } 4 \text {, uno será el } \\
\text { lanzador, otro el juez que validará el lanzamiento, el } \\
\text { tercero el juez técnico el cual dará la puntuación } \\
\text { sobre la ejecución técnica del lanzamiento y el cuarto } \\
\text { compañero, hará la función de medidor. Cada jugador } \\
\text { contará con tres lanzamientos. El profesor atenderá a } \\
\text { que ejecuten correctamente todos los roles que se } \\
\text { hayan de adoptar. }\end{array}$ \\
\hline $\begin{array}{l}\text { 7. Conocer y valorar la diversidad de } \\
\text { actividades físicas y deportivas y los } \\
\text { entornos en que se desarrollan, participando } \\
\text { en su conservación y mejora. }\end{array}$ & +++ & $\begin{array}{l}\text { Individualmente y en el menor tiempo posible, cada } \\
\text { jugador corriendo habrá de superar una serie de } \\
\text { obstáculos naturales (piedras, agujeros, arbustos,...) } \\
\text { de los que estará compuesto un circuito. }\end{array}$ \\
\hline $\begin{array}{l}\text { 8. Utilizar los recursos expresivos del } \\
\text { cuerpo y del movimiento para comunicar } \\
\text { sensaciones, ideas y estados de ánimo y } \\
\text { comprender mensajes expresados de este } \\
\text { modo. }\end{array}$ & + & $\begin{array}{l}\text { Por parejas, un jugador se colocará en una de las fases } \\
\text { del lanzamiento de disco y el otro con los ojos } \\
\text { vendados lo irá tocando hasta conseguir adivinar en la } \\
\text { fase en la que se encuentra. }\end{array}$ \\
\hline
\end{tabular}

Cuadro 1. Relación entre objetivos de área, significatividad y ejemplo de planteamiento lúdico de iniciación atlética.

Los contenidos han de tener una concepción más abier$\mathrm{ta}$, siendo potenciadores de las capacidades y el atletismo se ha de entender como un contenido que estará a disposición del profesor para conseguir las metas que persigue la educación a través de conceptos, procedimientos y actitudes. 


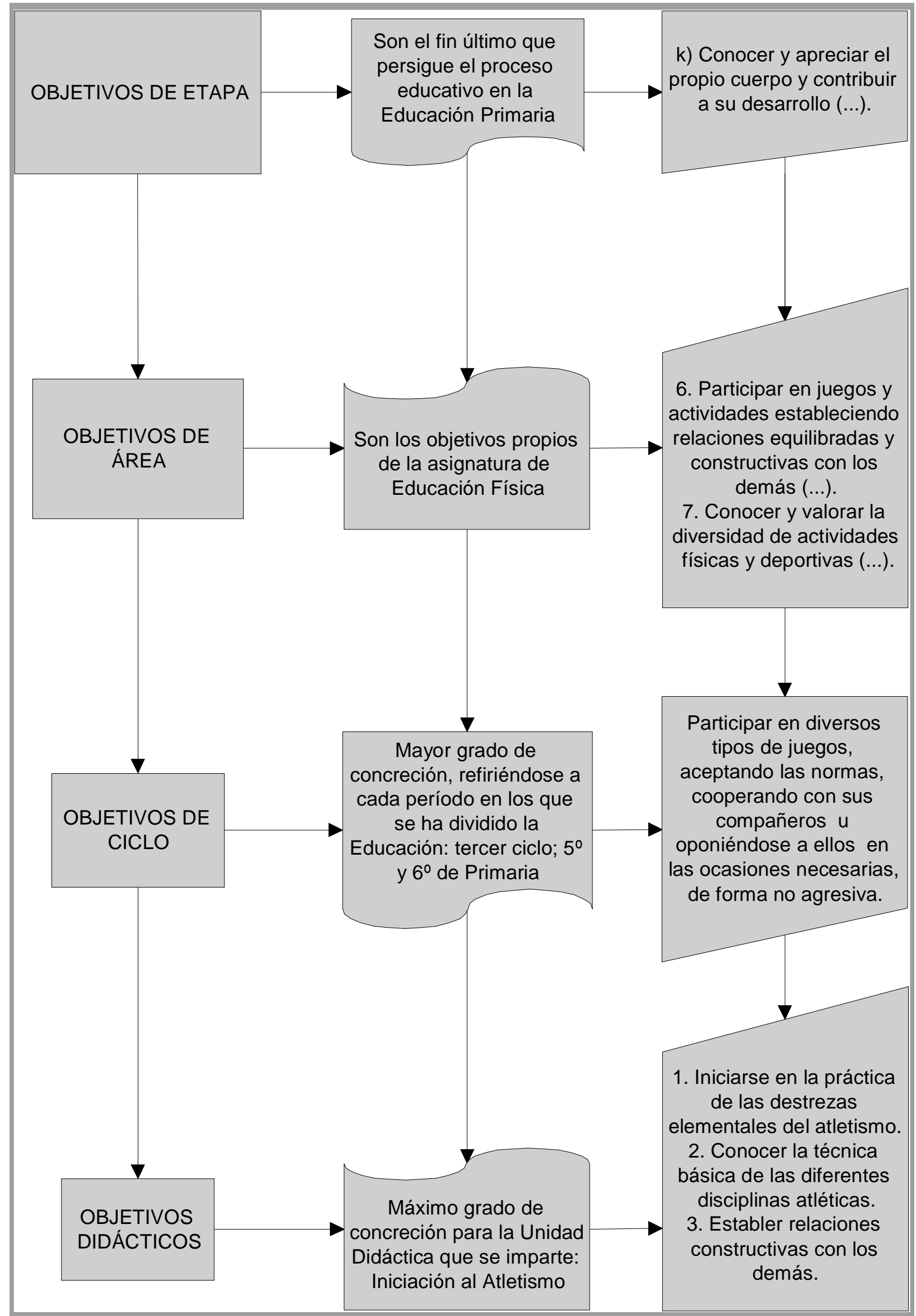

Cuadro 2. Relación entre los niveles de concreción de los objetivos y la iniciación del atletismo. 
Partiendo de los cinco bloques de contenidos existentes (el cuerpo: imagen y percepción; el cuerpo: habilidades y destrezas; el cuerpo: expresión y comunicación; salud corporal y los juegos), éstos hay que entenderlos como medios e instrumentos a través de los cuales se han de conseguir los objetivos, sin embargo, como ya apuntábamos anteriormente ni deporte ni atletismo aparecen como bloque de contenidos por lo que se hace necesario buscarle un lugar adecuado.

Autores como Parlebas (1976), o Blázquez (1986), se argumentan a favor del deporte como medio educativo, haciendo necesario minimizar la distancia que lo separa del juego, consiguiendo que se produzca una transición no traumática entre ambos, pues el juego es un excelente medio de iniciación deportiva (Díaz Suárez, 1996), y por tanto, extensible al atletismo, rompiendo con la idea de que los juegos son únicamente adecuados para transferir los aprendizajes a deportes colectivos, donde hay un predominio del componente táctico.

Se ha de abandonar la idea de que en el atletismo ha de primar el trabajo técnico mediante propuestas analíticas, dejando al juego como recurso a través del cual se libere la tensión acumulada por el trabajo rutinario y dejando de entender el juego como medio para mantener una motivación mermada por una práctica atlética falta de interés por sus practicantes.

El atletismo como contenido que persigue la educación del niño y la consecución de los objetivos del área podría formar parte de cualquiera de estos cinco bloques, pero teniendo en cuenta sus características y las aportaciones de autores especialistas en el campo ha de ubicarse dentro del "Los juegos".

or tanto, juego y atletismo deben ir enlazados dentro de este bloque de contenidos, centrándose principalmente en el tercer ciclo de la Educación Primaria para el desarrollo de la iniciación y la práctica deportiva, donde los juegos habrán de ir dando paso a los deportes por medio de los juegos reglados, minideportes, deportes simplificados, predeportes, etc., que son el nexo de unión entre el juego y el atletismo.

\section{Las orientaciones metodológicas}

Entendiendo por orientaciones metodológicas a todo el conjunto de directrices y enfoques didácticos que orientan al profesor en su tarea educativa $y$, de forma especial, en la selección y presentación de las actividades que realizan los alumnos en su proceso de aprendizaje (Díaz Lucea, 1995), las principales que encontramos en el Real Decreto (M.E.C., 1991a) se pueden concretar en las siguientes:

1. Enfoque globalizador, funcionalidad de los aprendizajes y actividad constructiva del niño. El alumno pondrá en práctica los nuevos conocimientos, asegurando una relación con la vida real. El aprendizaje habrá de ser vivenciado y global, atendiendo a las diferencias individuales, lo que dicho de otro modo, pondrá al alumno en el centro del eje del proceso de enseñanza - aprendizaje, siendo éste el verdadero protagonista de sus aprendizajes y dejando al profesor como mediador y guía, favoreciendo una práctica real con diferentes niveles de ejecución y de solución motriz.

2. Actividad lúdica como principal recurso. El juego será el elemento esencial de las propuestas metodológicas que se realicen para la práctica del atletismo dentro del marco educativo, rompiendo con planteamientos discriminatorios y favoreciendo una práctica coeducativa.

3. La evaluación será el punto de referencia para la actuación pedagógica, adecuando el proceso de enseñanza al progreso real del aprendizaje de los alumnos. El profesor jugará un papel importante para que se centre la motivación en la propia práctica atlética por medio del juego, ya que ésta resultará atractiva por sí misma con independencia de los logros deportivos que se consigan alcanzar.

\section{Criterios de Evaluación}

Según Contreras (1997), la evaluación supone un proceso por el cual se obtiene información que se utiliza para tomar decisiones o efectuar juicios, tanto sobre la enseñanza - aprendizaje, como sobre la estrategia docente empleada.

Bajo nuestra opinión una unidad didáctica basada en propuestas lúdicas a través de las cuales se les presenten a los alumnos el atletismo, constituyen un escenario ideal para observar y registrar la información referente a los aprendizajes orientados en los criterios de evaluación.

La Ley Orgánica General del Sistema Educativo (LOGSE), de ja claro que ya no sólo se evalúa el proceso de aprendizaje de los alumnos sino también el proceso de enseñanza (M.E.C., 1991a). La evaluación no se ha de limitar a comprobar la adquisición de los contenidos por parte de los alumnos en la iniciación al atletismo, sino que debe permitir sacar conclusiones sobre la adecuación o no de la unidad didáctica.

Esta evaluación del proceso de enseñanza la realiza el profesor reflexionando sobre su propia práctica docente en los aspectos referidos a si los objetivos han sido adecuados al nivel del grupo, si los contenidos de iniciación atlética han sido apropiados para la consecución de los objetivos propuestos, si las actividades han estado secuenciadas y han sido atrayentes, si el tiempo ha sido suficiente, etc.

Por tanto, a modo de conclusión, se puede afirmar que la iniciación al atletismo tiene sentido en la Educación Primaria desde una concepción educativa hacia el final de etapa (especialmente quinto y sexto de Primaria), donde las disciplinas atléticas se presentan como medio para el desarrollo de las capacidades del niño, teniendo en consideración una serie de características que se han de cumplir para alcanzar los objetivos planteados como son: enseñanza globalizada, fomento de las relaciones con los demás, adaptación a sus intereses y necesidades, afán de superación y actividad lúdica y motivante. 


\section{Ejemplo de unidad didáctica: Iniciación al atletismo}

A modo de recapitulación se considera pertinente en este apartado exponer un ejemplo aséptico y descontextualizado de las peculiaridades propias de un centro educativo concreto, pero que sirva de propuesta donde se plasmen todos los aspectos abordados hasta el momento tales como el atletismo y su iniciación, los objetivos, etc. y también sirva de modelo sobre el cual se puedan construir verdaderas unidades didácticas donde se tengan en cuenta las características concretas del centro, de los alumnos, de la disponibilidad de material y recursos, etc.

De tal manera, se ha elaborado una ficha de unidad didáctica perteneciente a una imaginaria programación de aula, donde se recogen los objetivos didácticos, los contenidos conceptuales, actitudinales y procedimentales, la metodología y la evaluación (cuadro 3), persiguiendo con ello, ser el colofón de la justificación de la iniciación al atletismo dentro del currículum de Educación Física para la Educación Primaria.

\begin{tabular}{|c|c|c|c|c|c|c|c|c|}
\hline \multicolumn{9}{|c|}{ UNIDAD DIDÁCTICA: INICIACIÓN AL ATLETISMO } \\
\hline \multicolumn{4}{|c|}{ ¿QUÉ ENSEÑAR? } & \multirow{2}{*}{\multicolumn{3}{|c|}{$\begin{array}{c}\text { ¿CÓMO Y CUANDO ENSEÑAR? } \\
\text { METODOLOGÍA }\end{array}$}} & \multirow{2}{*}{\multicolumn{2}{|c|}{$\begin{array}{l}\text { ¿QUÉ, CÓMO Y CUANDO EVALUAR? } \\
\text { EVALUACIÓN APRENDIZAJE }\end{array}$}} \\
\hline \multirow{2}{*}{$\begin{array}{l}\text { OBJETIVOS } \\
\text { DIDÁCTICOS }\end{array}$} & \multicolumn{3}{|c|}{ CONTENIDOS } & & & & & \\
\hline & Conceptuales & Actitudinales & Procedimentales & Actividades & Material y recursos & Estrategia & Criterios & Instrumentos \\
\hline \multirow{4}{*}{$\begin{array}{l}1^{\circ} \text { : Iniciarse en } \\
\text { la práctica de } \\
\text { las destrezas } \\
\text { elementales del } \\
\text { atletismo. } \\
2^{\mathrm{a}} \text { : Conocer la } \\
\text { técnica básica } \\
\text { de las } \\
\text { diferentes } \\
\text { disciplinas } \\
\text { atléticas. } \\
3^{\mathrm{a}} \text { : Desarrollar } \\
\text { a partir de una } \\
\text { forma jugada, } \\
\text { toda una serie } \\
\text { de prácticas } \\
\text { atléticas. } \\
4^{\mathrm{a}} \text { : Establecer } \\
\text { relaciones } \\
\text { constructivas } \\
\text { con los demás. }\end{array}$} & \multirow{4}{*}{$\begin{array}{l}\text { Conocimiento de } \\
\text { la técnica básica } \\
\text { de los } \\
\text { desplazamiento } \\
\text { s, los saltos y los } \\
\text { lanzamientos. } \\
\text { Comprensión de } \\
\text { las coordinacio- } \\
\text { nes dinámicas } \\
\text { generales con } \\
\text { implementos y } \\
\text { el proceso de } \\
\text { lateralización y } \\
\text { ambidextrismo } \\
\text { para posibilitar } \\
\text { un mejor } \\
\text { asentamiento } \\
\text { del aprendizaje } \\
\text { en el atletismo. }\end{array}$} & \multirow{4}{*}{$\begin{array}{l}\text { Interés por la } \\
\text { práctica } \\
\text { atlética } \\
\text { aceptando los } \\
\text { diferentes } \\
\text { niveles de } \\
\text { destreza, tanto } \\
\text { en sí mismo } \\
\text { como en los } \\
\text { otros. } \\
\text { Respeto a los } \\
\text { compañeros y } \\
\text { al material. } \\
\text { Aceptación de } \\
\text { las normas y } \\
\text { reglas del } \\
\text { atletismo y del } \\
\text { papel que } \\
\text { corresponda } \\
\text { desempeñar } \\
\text { como jugador. }\end{array}$} & \multirow{4}{*}{$\begin{array}{l}\text { Práctica } \\
\text { adaptada de la } \\
\text { marcha atlética } \\
\text { y de las } \\
\text { carreras, de los } \\
\text { lanzamientos y } \\
\text { de los diferentes } \\
\text { saltos. } \\
\text { Utilización de } \\
\text { reglas para la } \\
\text { organización de } \\
\text { situaciones } \\
\text { colectivas de } \\
\text { juego y de } \\
\text { estrategias } \\
\text { básicas. } \\
\text { Aumento de la } \\
\text { precisión y } \\
\text { economía de las } \\
\text { habilidades. }\end{array}$} & \multirow{4}{*}{$\begin{array}{l}1^{a} \text { sesión: } \\
\text { Detección del } \\
\text { dominio y } \\
\text { desarrollo de } \\
\text { habilidades. } \\
2^{a}, 3^{a} \text { y } 4^{a} \text { sesión: } \\
\text { Propuestas } \\
\text { lúdicas de } \\
\text { actividades } \\
\text { relacionadas con } \\
\text { los } \\
\text { desplazamientos. } \\
5^{a}, 6^{a} \text { y } 7^{a} \text { sesión: } \\
\text { Propuestas } \\
\text { lúdicas de } \\
\text { actividades } \\
\text { relacionadas con } \\
\text { los saltos. } \\
8^{a}, 9^{a} \text { y } 10^{a} \text { sesión: } \\
\text { Propuestas } \\
\text { lúdicas de } \\
\text { actividades } \\
\text { relacionadas con } \\
\text { los lanzamientos. } \\
11^{a} \text { sesión: } \\
\text { Competición por } \\
\text { equipos y } \\
\text { evaluación. }\end{array}$} & \multirow{4}{*}{$\begin{array}{l}\text { Pelotas de } \\
\text { diferentes tamaños, } \\
\text { picas, colchonetas } \\
\text { quitamiedos, } \\
\text { simuladores de } \\
\text { peso, vallas, conos, } \\
\text { bancos suecos, } \\
\text { globos, pelotas de } \\
\text { espuma, elásticos, } \\
\text { cuerdas, pandero, } \\
\text { aros de diferentes } \\
\text { tamaños, testigos, } \\
\text { balonces } \\
\text { medicinales, } \\
\text { saltómetros, } \\
\text { trampolin, cinta } \\
\text { adhesiva y todo tipo } \\
\text { de material } \\
\text { alternativo. }\end{array}$} & \multirow[t]{4}{*}{$\begin{array}{l}\text { Mixta, } \\
\text { empleando } \\
\text { el juego } \\
\text { como } \\
\text { elemento } \\
\text { conductor y } \\
\text { motivador, al } \\
\text { que se } \\
\text { modifica } \\
\text { introducién- } \\
\text { dole una } \\
\text { serie de } \\
\text { reglas para } \\
\text { iniciarles en } \\
\text { los aspectos } \\
\text { técnicos del } \\
\text { atletismo. }\end{array}$} & $\begin{array}{l}\text { Si practica las } \\
\text { destrezas } \\
\text { elementales del } \\
\text { atletismo. } \\
\text { Si conoce la } \\
\text { técnica básica de } \\
\text { las diferentes } \\
\text { disciplinas } \\
\text { atléticas. } \\
\text { Si establece } \\
\text { relaciones } \\
\text { constructivas con } \\
\text { los demás. }\end{array}$ & $\begin{array}{l}\text { Observación } \\
\text { sistemática. } \\
\text { Corrección de } \\
\text { actividades. } \\
\text { Trabajo en } \\
\text { grupos: } \\
\text { propuestas } \\
\text { lúdicas. }\end{array}$ \\
\hline & & & & & & & \multicolumn{2}{|c|}{ EVALUACIÓN ENSEÑANZA } \\
\hline & & & & & & & Criterios & Instrumentos \\
\hline & & & & & & & $\begin{array}{l}\text { Si los objetivos } \\
\text { han sido } \\
\text { adecuados al } \\
\text { nivel del grupo y } \\
\text { las actividades } \\
\text { motivantes. }\end{array}$ & $\begin{array}{l}\text { Cuestionario } \\
\text { acerca del interés } \\
\text { por la práctica del } \\
\text { atletismo. }\end{array}$ \\
\hline
\end{tabular}

\section{Bibliografía}

Arnold, P.J. (1991). Educación física, movimiento y currículum. Morata. Madrid.

Blázquez, D. (1986). Iniciación a los deportes de equipo. Martínez Roca. Barcelona.

Castejón, F.J. (1997). La iniciación deportiva en la Educación Primaria: lo que opinan los profesores de Educación Física. Apunts: Educación Física y Deportes. 48, 24-33.

Castejón, F.J. y López, V. (1997). Iniciación deportiva. En Castejón, F.J. Manual del Maestro Especialista en Educación Física. Pila Teleña. Madrid.

Contreras, O. (1997). Didáctica de la Educación Física. En Castejón, F.J. Manual del Maestro Especialista en Educación Física. Pila Teleña. Madrid.

Contreras, O.R. (1998). Didáctica de la Educación Física. Un enfoque constructivista. Inde. Barcelona.

De la Torre, E. y Hernández, J.L. (1996). La iniciación deportiva y los elementos curriculares del área de Educación Física en Primaria. (Decreto de la Comunidad
Autónoma Andaluza). En Díaz Suárez, A. El deporte en educación primaria. DM. Murcia.

Díaz Lucea, J. (1995). El currículum de la Educación Física en la Reforma Educativa. Inde. Barcelona.

Díaz Suárez, A. (1996). El deporte en educación primaria. DM. Murcia.

Giménez, F.J. (1997). El deporte como contenido de Educación Física. En Giménez, J., Sáenz-López, P. y Díaz Trillo, M. El deporte escolar. Universidad de Huelva.

Parlebas, P. (1976). Activités physiques et éducation motrice. Revista EPS. París.

Real Decreto 1006/1991, de 14 de junio, por el que se establece las enseñanzas mínimas correspondientes a Educación Primaria. BOE, n 152 y suplemento. 26 de junio de 1991. Madrid.

Real Decreto 1344/1991, de 6 de septiembre, por el que se establece el currículo de la Educación Primaria en el área de gestión del MEC. BOE $n^{\circ} 220.13$ de septiembre de 1991. Madrid.

Rodríguez, J. (1993). Desarrollo curricular de Educación Física para Enseñanza Primaria. Escuela Española. Madrid.

Zurita, F. (1998). Deportes Individuales en Primaria. Atletismo y Tenis. Proyecto Sur. Granada. 